\title{
Wood piece quality evaluation using Choquet integral and fuzzy merging
}

\author{
Jeremy Jover ${ }^{1,2}$, Vincent Bombardier ${ }^{1,2}$ and Andre Thomas ${ }^{1,2}$ \\ ${ }^{1}$ Université de Lorraine, CRAN, UMR 7039, Campus Sciences, BP 70239, \\ Vandouvre-lès-Nancy Cedex, 54506, France \\ ${ }^{2}$ CNRS, CRAN, UMR 7039, France \\ $\{$ jeremy.jover, vincent.bombardier, andre.thomas\}@univ-lorraine.fr
}

\begin{abstract}
Keywords: Virtual Quality, RX Computed Tomography, Information Fusion,fuzzy operators, Choquet integral, divergent process

Abstract: $\quad$ This paper presents a way to evaluate the wood product quality according to his tomographic image. The use of $\mathrm{X}$ ray computed tomography and ad-hoc software allows to have a representation of an item (a product) before the first cutting operation. From this representation, we aim to obtain an honest evaluation of their aesthetic quality. To do that singularity features are extracted and their impact on the product visual quality is assesed thanks to the Choquet integral. Next, the visual quality is evaluated by merging singularity impacts and singularity number criterion using suitable operators. Three operators are compared to the mean operator which is the commonly used one when there are few knowledge on the decision process.
\end{abstract}

\section{INTRODUCTION}

Divergent processes have always presented a difficulty for the traceability implementation. The raw material cutting leads two problems:

- The link between material and production information is difficult to establish and to maintain all along the product cycle life.

- Foresee the finish products features, which are very useful for traceability and production management, is full of uncertainty.

Some solutions are proposed to overcome this problem especially in the food industry. These solutions are based on marking and documenting batches (Dupuy, 2004). However a part of the root information is lost and an unique identification is not still possible (what is ideally expected).

The wood industry is also concerned by these divergent process problems. From a tree, products satisfying the end customer's needs must be produced. Moreover the product origin traces have to be conserved for traceability reasons (PEFC1, 2010). The wood, being a heterogeneous material, increases complexity. Structurally, the wood is composed of aligned fibres following a longitudinal axe. It not reacts in the same way following the different axes (longitudinal, radial, tangential). The wood colour is not homogeneous too: the growing rings, the singularity presence or the fungal attacks (blue stain) create heterogeneity on visual and mechanical points of view.

In sawmills, the optimization, in order to have the right products, is an important and complicated task. Sawyers have to saw products which have characteristics needed by customers, from a raw material which internal characteristics are unknown. Dimensionally, it is easy to foresee and have the right product dimension (apply the cutting pattern), but other features as the colour or the mechanical resistance are more complicated to estimate and to characterize before the log is sawed due to their subjectivity character and the wood heterogeneity.

Our researches is concerned by the information loss reduction in the wood industry. We have proposed a solution to mark and maintain the origin information of the trees (Jover et al., 2011). In this study, we propose a way to determine the wood product characteristics before sawing operation in order to satisfy the customer needs and the optimal determination of the production element (net requirement for each product quality class). The proposed approach aims to automate the product qualification process (quality product estimation) usually done by an operator. The global process is described Fig. 1.

In this article, we shortly present the wood quality notion, the sawing optimization process and the virtual sawing concept allowing to extract the virtual product. Then we explain how we characterise sin- 
gularities and calculate their impact on product with the Choquet integral. Impact calculation is improved by using learning process to calculate the Choquet capacities. To finish, we describe a way to estimate the wood products quality by using the impact singularities. We demonstrate the feasibility with distinguish wood pieces.

\section{PRODUCTION FORESEE IN SAWMILL}

\subsection{The visual quality of wood products}

Concerning the needs of the first transformation customers ${ }^{1}$, there are three kinds of quality: the dimensional quality, the mechanical quality and the visual (aesthetic) quality. The dimensional quality is easy to characterize (dimension piece precision). The mechanical quality is more skillful to evaluate. The clear wood has a mechanical resistance which can be reduced by the singularities presence (knots, crack, rot ). Techniques based on the vibrations give results as explained in (Guillot et al., 1996).

The last one is the visual quality. It is the most complicated to evaluate because the visual quality is a subjective decision. The visual quality is defined in the standard NF EN 1611. This standard defines different classes of qualities based on the singularity features measurement (size, numbers, type,...). But the evaluation is done by a Human Expert which has to estimate the quality within a short time (according to the sawmill high production rate). In this short time, the expert cannot evaluate singularity features as precisely as the standard define them. So standard definition is not adapted to the evaluation. More over the wood is "intrinsically fuzzy" (Bombardier et al., 2007). Boundaries between clear wood and singularities are not so easy to determine and impact the characteristic measures. A big part of the price is based on the quality, so its determination is important for customer and sawyers.

\subsection{Raw material optimization}

Sawyers optimise wood by estimating which cutting plan allows to have the best material yield and satisfy the customer requirement. That is why it is essential to foresee product features which would be cut in the $\log$.

\footnotetext{
${ }^{1}$ The first transformation of the wood is relative to the first steps of the wood value chain; that is to say, activities from the living tree in the forest to the output of the sawmill.
}

The Expert (present at the optimization post) estimates product features (dimension, mechanical resistance, visual quality) according to the external log features and his experiment. He is able to determine approximately which defects are present in the wood (according to the external log features) and decides which cutting plan is the most appropriate to obtain the customer requirement. So final product aspect and quality are more or less well determined. We easily understand that all singularities are not visible on the surface and singularities which are visible give only incomplete information on their shapes in wood. Lot of researches have proposed solutions taking into account the external shapes of logs (Todoroki and Rönnqvist, 2002). But these optimisations are only based on the log dimensional features and do not take into account the visual and mechanical characteristics. This paper proposed a way (virtual sawing) to address this issue.

\subsection{Virtual sawing}

The use of non destructive control techniques $(\mathrm{Bu}-$ cur, 2003), in particular X ray computed tomography, allows to have a $3 \mathrm{D}$ representation of the $\log$ (internal and external) to be cut. (Almecija et al., 2012) use volumetric information to improve part log quality determination and their sorting. In our proposition we investigate this step and the global process is described in the figure 1.

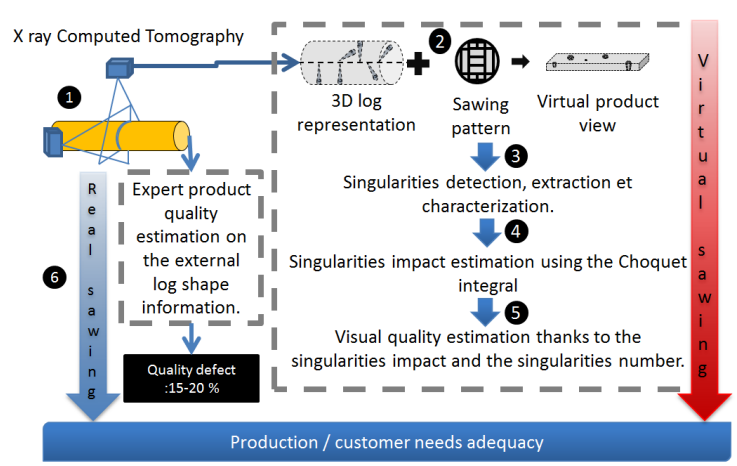

Figure 1: Extraction and exploitation process of virtual products.

The log representation is virtually sawed with an ad-hoc software according to a cutting plan. This leads to obtain a numerical view of all product faces which should be obtained. The figure 2 shows one face for one product. The obtained image represents the face of a product according to the density data. Some information cannot be obtained (the colour) and the distinction between detected object is not so easy. 
All of these add imperfection, imprecision and uncertainty and make the quality determination harder.

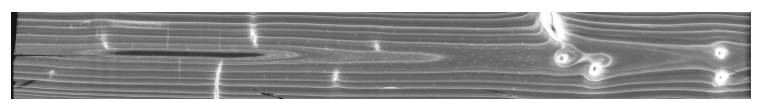

Figure 2: Example of a face extracted with the virtual sawing

\subsection{Problematic}

Our aim is to propose a process to estimate the wood product quality according to the face picture extracted by the virtual sawing stage. We consider that the singularity features are computed in a similar way as (Bombardier et al., 2007). So we propose a way to determine quality products from these measurements. As obtained information is uncertain, incomplete and imprecise, we use methods allowing taking into account this imperfection especially the Choquet Integral and fuzzy fusion operators. In this paper, we decide to estimate the singularity impact on the product and then to determine the piece quality.

\section{SINGULARITY IMPACT EVALUATION}

\author{
$L_{p} \quad$ Product length \\ $l_{p} \quad$ Product width \\ $I_{p} \quad$ Product grey level \\ $T_{s} \quad$ Singularity size \\ $X_{s} \quad$ Singularity position on product length \\ $Y_{S} \quad$ Singularity position on product width \\ $I_{S} \quad$ Singularity grey level
}

\subsection{Singularity criterion measurement}

In our study, we evaluate the visual quality of the wood. So the criteria have to reflect singularity impact on the visual quality. (Almecija et al., 2012) defines forty criteria to evaluate quality. From this forty criteria, only around twenty concern the final product and only a dozen the visual quality. To evaluate singularity impact on density data, we only use the four of them which are measurable on a grey scale image.

The first criterion $u_{t}$, described by the equation (1), reflects the singularity size. More a singularity is big, more the visual quality is down grading. Moreover the expert judgement stipulates a singularity higher than $50 \mathrm{~mm}$ is considered as highly critical.

$$
\left\{\begin{array}{l}
\text { If } l_{p} \leq 50 \mathrm{~mm}, u_{t}=1-\frac{T_{s}}{l_{p}} \\
\text { If } l_{p}>50 \mathrm{~mm} \text { and } T_{s} \leq 50 \mathrm{~mm}, u_{t}=1-\frac{T_{s}}{50} \\
\text { If } l_{p}>50 \mathrm{~mm} \text { and } T_{s}>50 \mathrm{~mm}, u_{t}=0
\end{array}\right.
$$

The second criterion $u_{x}$, described by the equation (2), reflects the position of the singularity on the product length. More a singularity is close to the product end, more this singularity lost importance and the quality becomes higher.

$$
u_{x}=\frac{\left|L_{p} / 2-X_{s}\right|}{L_{p} / 2}
$$

The third criterion $u_{y}$, described by the equation (3), reflects the position of the singularity on the product width. More a singularity is close to the product edge, more this singularity lost important and the quality becomes higher.

$$
u_{y}=\frac{\left|l_{p} / 2-Y_{s}\right|}{l_{p} / 2}
$$

The last criterion $u_{c}$, described by the equation (4), reflects contrast between the singularity and the product background. More the contrast is weak, less this singularity is visible and more the quality increases.

$$
u_{c}=1-\frac{\left|I_{s}-I_{p}\right|}{I_{p}}
$$

In the following part, we proposed a method using Choquet integral according to the fact that the singularity characteristic measurements are full of imperfection and imprecision (see section 2).

\subsection{Impact calculation using the Choquet integral}

The Choquet integral was proposed by Gustave Choquet in 1954 (Choquet, 1953). Their use in the multi-criteria decision making domain appears in the nineties in different context (car industry, strategical placement, ) and similar classification problems are usually process with the Choquet integrals (Grabisch and Labreuche, 2008). They allow taking into consideration importance of each criterion and the interactions existing between each of them.

Let $\{X\}:\left\{x_{1}, \ldots, x_{n}\right\}$ be a set of normalized criteria, consider a capacity $\mu: \wp(X) \rightarrow[0,1]$ on this set, verifying:

$$
\left\{\begin{array}{l}
\mu(\emptyset)=0 \\
\mu(X)=1 \\
\mu(A)<\mu(B), \forall A \subset B \text { and } \forall B \subset X
\end{array}\right.
$$


The capacity defines all weights and interactions. Then Choquet integral is defined as follow:

$$
C_{\mu}\left(u_{1}, \ldots, u_{n}\right)=\sum_{i=1}^{n}\left(u_{\sigma k}-u_{\sigma k-1}\right) \mu\left(A_{\sigma k}\right)
$$

Where $\sigma$ is the indices permutation satisfying:

$$
\begin{aligned}
& 0=u_{\sigma 0} \leq u_{\sigma 1} \leq \ldots \leq u_{\sigma n-1} \leq u_{\sigma n} \leq 1 \\
& u_{\sigma 1}=\operatorname{Min}\left(u_{i}\right) \text { et } u_{\sigma n}=\operatorname{Max}\left(u_{i}\right)
\end{aligned}
$$

and $A_{\sigma k}=\left\{g_{\sigma k}, \ldots, g_{\sigma n}\right\}$ the features non used in previous steps.

In our case, $C_{\mu}(u)$ corresponds to the measure of the singularity impact on the product when the Choquet integral is apply on the criteria $\left(\mu_{i}\right)$. More the value tends to 1 , less the singularity is important (our own standards). The Choquet integral is useful when the knowledge and the learning batches are low. The greatest challenge is the definition of the capacities (Grabisch et al., 2008). To do so, some approaches were developed to learn the capacities. We detail them in the next subsection.

\subsection{Learning process of the capacities}

In order to have a better definition of the capacities used in the Choquet integral, we decide to use a learning process. Different approaches can be used to identify capacities (Grabisch et al., 2008):

- The Least Square approach (LS): based on the expert knowledge on each element. The expert attributes a target impact value to each element (experted value) in the learning lot and system searches capacities values that minimise the difference square between the computed value and the expected value.

$$
\operatorname{MinF}_{L S}(\mu):=\sum_{x \in O}\left[C_{\mu}(u(x))-y(x)\right]^{2}
$$

- The Linear Programming approach (LP): proposed by Marichal and Roubens in (Marichal and Roubens, 2000), it is based on the expert knowledge on the global ranking of the batch elements. The approach looks for the value which satisfy as closely as possible the ranking establish by the expert.

$$
\begin{aligned}
& \operatorname{MaxF}_{L P}(\varepsilon):=\varepsilon \\
& \text { subj. to }\left\{\begin{array}{l}
\sum_{T \subseteq S} m_{v}(T \cup i) \geq 0, \forall i, \forall S \\
\sum_{T \subseteq N} m_{v}(T)=1 \\
C_{v}(u(A))-C_{v}(u(B)) \geq \delta_{C} \\
\vdots
\end{array}\right.
\end{aligned}
$$

In (Grabisch et al., 2008), authors explain that the least square approach is appropriate when it is possible to attribute precisely the desired value. They explain too that the linear programming is better for cases which it is easy to give a pre-order between the learning lot element. This is our case for the evaluation of the singularity impact because it is hard for the expert to give a score for each singularities (due to number and variation of the cases). The expert decides of a pre-order between elements composing the learning batch (with a $\delta_{C}$ corresponding to the minimum margin to respect the ranking). This constraint, noted E, can be translate by:

$$
\begin{aligned}
& C_{\mu}(a)>C_{\mu}(b)>\ldots>C_{\mu}(k) \\
& \text { with } C_{\mu}(u(i)) \geq C_{\mu}(u(i+1))+\delta_{C}
\end{aligned}
$$

Some conditions can be imposed, over the element pre-order, on the criterion importance and/or interaction. The expert can expressed the criterion importance against one another. By the used of the Shapley indices $\phi$ (which indicates the global importance of each criterion), the expert expresses the equality between two criteria. The value $\delta_{\phi}$ is the maximal distance between two Shapley values to consider two criteria are equal. This constraint, noted $\mathrm{S}$, can express for a couple of criteria A and B as:

$$
-\delta_{\phi} \leq \phi_{v}\left(\mu_{A}\right)-\phi_{v}\left(\mu_{B}\right) \leq \delta_{\phi}
$$

More over the Expert can express constraints on the interaction between the criteria. The interaction between two criteria can be easily expressed by the expert because the phenomena is understandable.But the interaction between more than two criteria is harder to understand and express. The last condition (apply on the interaction indices) is only expressed on interaction between a pair of criteria. This constraint, noted I, can be:

- negative (redundancy) : $I_{V}(\{A, B\})<0-\delta_{I}$

- positive (synergies) : $I_{V}(\{A, B\})>0+\delta_{I}$

- null (no interaction) : $I_{V}(\{A, B\})=0 \pm \delta_{I}$

$\delta_{I}$ is the minimum threshold value in absolute value to consider the interaction as significant.

Using the software $\mathrm{R}$ and the Kappalab $\mathrm{R}$ package, we compute the LP approach in order to determine the capacities and influence of the calculated values on the Choquet integral results.

\subsection{Result of the learning process LP}

The learning batch described in the table 1, is composed of singularities which are commonly found in the wood.

Expert constraints are describe below: 
Table 1: Learning batch.

\begin{tabular}{c||c|c|c|c} 
Singularity & $\mathrm{T}$ & $\mathrm{X}$ & $\mathrm{Y}$ & $\mathrm{C}$ \\
\hline $\mathrm{a}$ & 0.75 & 0.72 & 0.2 & 0.93 \\
$\mathrm{~b}$ & 0.41 & 0.09 & 0 & 0.98 \\
$\mathrm{c}$ & 0.39 & 0.27 & 0.24 & 0.9 \\
$\mathrm{~d}$ & 0.69 & 0.36 & 0.8 & 0.37 \\
$\mathrm{e}$ & 0.42 & 0.75 & 0.32 & 0.3 \\
$\mathrm{f}$ & 0.75 & 0.81 & 0.52 & 0.09
\end{tabular}

- On the elements batch (E): The singularities are ranked as they are stored in the table 1 from the best to the worst with $\delta_{C}=0.05$.

- On the importance criterion (S): criteria $\left[\mu_{t}, \mu_{c}\right]$ have the same importance, criteria $\left[\mu_{x}, \mu_{y}\right]$ too and criteria $\left[\mu_{t}, \mu_{c}\right]$ are more important than $\left[\mu_{x}, \mu_{y}\right]$ $\left(\delta_{\phi}=0.1\right): \phi_{v}\left(\mu_{t}\right)=\phi_{v}\left(\mu_{c}\right)>\phi_{v}\left(\mu_{x}\right)=\phi_{v}\left(\mu_{y}\right)$.

- On the influence among criterion (I): criteria $\left[\mu_{t}, \mu_{c}\right]$ are in synergy and $\left[\mu_{x}, \mu_{y}\right]$ too $\left(\delta_{I}=0.05\right)$ : $I_{v}\left(\left\{\mu_{t}, \mu_{c}\right\}\right)>0$ and $I_{v}\left(\left\{\mu_{x}, \mu_{y}\right\}\right)>0$.

The results of the Choquet value, the Shapley values and the interaction indices are respectively presented in the table 2, 3 and 4 . In table2, the second column corresponds to values obtained without any importance( singleton capacity equal to 0.25 ) and constraints (other capacity equal to the sum of the singleton capacities) between criteria.

Table 2: Results for the LP approach for the different constraints.

\begin{tabular}{c||c|c|c|c} 
Singularity & $\emptyset$ & $\mathrm{E}$ & $\mathrm{E}+\mathrm{S}$ & $\mathrm{E}+\mathrm{S}+\mathrm{I}$ \\
\hline $\mathrm{a}$ & 0.65 & 0.878 & 0.878 & 0.873 \\
$\mathrm{~b}$ & 0.37 & 0.815 & 0.815 & 0.799 \\
$\mathrm{c}$ & 0.45 & 0.753 & 0.753 & 0.738 \\
$\mathrm{~d}$ & 0.55 & 0.450 & 0.569 & 0.593 \\
$\mathrm{e}$ & 0.46 & 0.387 & 0.422 & 0.532 \\
$\mathrm{f}$ & 0.49 & 0.325 & 0.359 & 0.471
\end{tabular}

Table 3: Shapley indices for the different constraints.

\begin{tabular}{c||c|c|c|c} 
Shapley value & $\mathrm{T}$ & $\mathrm{X}$ & $\mathrm{Y}$ & $\mathrm{C}$ \\
\hline $\mathrm{E}$ & 0.152 & 0.117 & 0.069 & 0.662 \\
$\mathrm{E}+\mathrm{S}$ & 0.397 & 0.074 & 0.045 & 0.484 \\
$\mathrm{E}+\mathrm{S}+\mathrm{I}$ & 0.322 & 0.169 & 0.098 & 0.411
\end{tabular}

Imposed Constraints are respected at each steps (E, E+S,E+S+I). We can see in the table 2 the order of the singularity is the same as the expert ranking.

The first constraint (based on the elements ranking) accords lot of importance to the contrast (Shapley value $\phi_{v}\left(\mu_{c}\right)=0.762$ ) and few on the other criteria. Moreover interaction between the criteria [T $\mathrm{C}]$ is negative (that is not corresponding to the expert choice). The figure 3 shows the variation of the Choquet integral value function of [X Y]. The criterion $\mathrm{Y}$ have few influence except up to 0.75 . This translate an expert view: the singularity position on the width
Table 4: Interaction indices for the different constraints (symmetric matrix).

\begin{tabular}{|c||c|c|c|c|}
\hline \multicolumn{5}{|c|}{$\downarrow$ constraints: $\mathrm{E} \downarrow$} \\
\hline Interaction & T & X & Y & C \\
\hline T & NA & 0.006 & -0.138 & -0.119 \\
X & & NA & 0.131 & -0.137 \\
Y & & & NA & 0.007
\end{tabular}

\begin{tabular}{c||c|c|c|c|}
\hline \multicolumn{5}{|c|}{$\downarrow$ constraints: $\mathrm{E}+\mathrm{S} \downarrow$} \\
\hline Interaction & $\mathrm{T}$ & $\mathrm{X}$ & $\mathrm{Y}$ & $\mathrm{C}$ \\
\hline \hline $\mathrm{T}$ & NA & -0.133 & -0.090 & 0.113 \\
$\mathrm{X}$ & & NA & 0.179 & -0.133 \\
$\mathrm{Y}$ & & & NA & -0.090
\end{tabular}

\begin{tabular}{c||c|c|c|c}
\hline \multicolumn{5}{|c}{$\downarrow$ constraints: $\mathrm{E}+\mathrm{S}+\mathrm{I} \downarrow$} \\
\hline Interaction & $\mathrm{T}$ & $\mathrm{X}$ & $\mathrm{Y}$ & $\mathrm{C}$ \\
\hline \hline $\mathrm{T}$ & NA & -0.219 & 0.032 & 0.050 \\
$\mathrm{X}$ & & NA & 0.050 & -0.285 \\
$\mathrm{Y}$ & & & NA & -0.226
\end{tabular}

upgrade the singularity only when it is very close to the side.

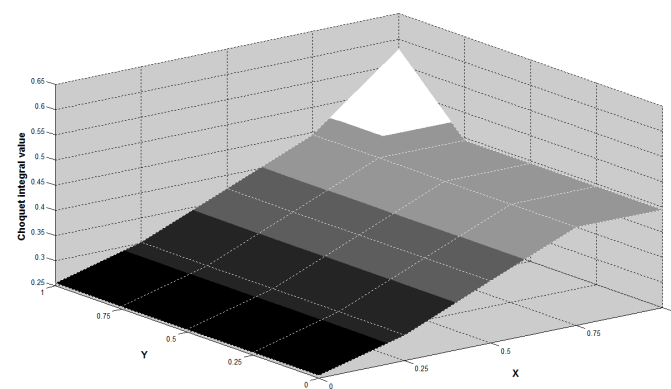

Figure 3: Influence of the $\mathrm{X}$ and $\mathrm{Y}$ criteria modification on the Choquet value with the weights obtained under $\mathrm{E}$ constrain and no variation of the $\mathrm{T}$ and $\mathrm{C}$ criteria $(u=$ $(0.75, \triangle(X), \triangle(Y), 0.09))$.

The addition of the constraint S (on Shapley indices) offers a positive interaction between [T,C] but not on $[\mathrm{X}, \mathrm{Y}]$. Moreover the importance of the criteria $[\mathrm{X}, \mathrm{Y}]$ are so little that they have few influence on the Choquet value. The figure 4 shows the Choquet value variation function of $\mathrm{X}$ and $\mathrm{Y}$ variation for a singularity $(u=(0.75, \triangle(X), \triangle(Y), 0.09))$. The criteria have no action when theirs values are bellow 0.75 . This comportment means that singularity position is important only when a singularity is close to the end and the side of the product.

The addition of the last constraint gives capacities which allow the respect of the constraints given by the expert. Moreover all criteria have impact on the calculation result. The figure 5 translates the $[\mathrm{X}, \mathrm{Y}]$ impact. Thereafter we use this weight for the product quality estimation. 


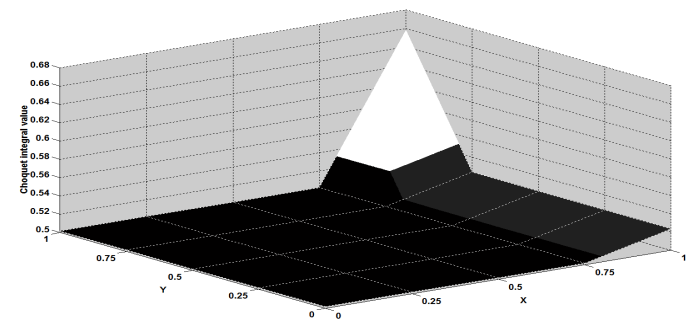

Figure 4: Influence of the $\mathrm{X}$ and $\mathrm{Y}$ criteria modification on the Choquet value with the weights obtained under E+S constrains and no variation of the $\mathrm{T}$ and $\mathrm{C}$ criteria $(u=$ $(0.75, \triangle(X), \triangle(Y), 0.09))$.

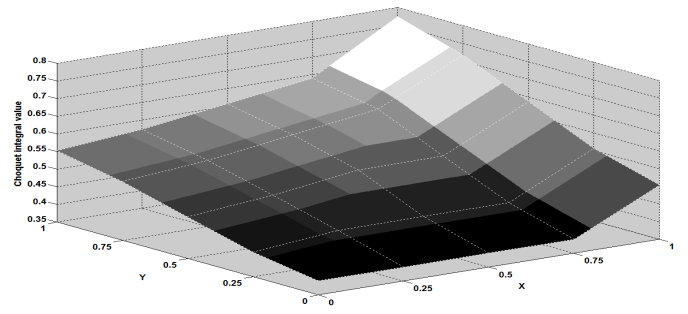

Figure 5: Influence of the $\mathrm{X}$ and $\mathrm{Y}$ criteria modification on the Choquet value with the weights obtained under $\mathrm{E}+\mathrm{S}+\mathrm{I}$ constrains and no variation of the $\mathrm{T}$ and $\mathrm{C}$ criteria $(u=$ $(0.75, \triangle(X), \triangle(Y), 0.09))$.

\section{PRODUCT QUALITY EVALUATION}

Once singularity impacts are determined, an estimation of the quality product is evaluated by merging these impact and the criterion evaluating the singularity number. In this part, we describe this criterion and the fusion operation.

\subsection{Singularity number criterion}

In the quality evaluation, the number of singularities is important. A product with a lot of singularities is more down grading because the clear wood homogeneity is broken.

To evaluate the number, we used the criterion $R_{n b}$ defined by (12). This criterion represents the expert vision: more there are singularities, more the product is down grading. Moreover when the number reaches towards a particular value, the criterion reaches towards 0 . We choose to used an exponential function. Following the particular number of singularities fixed by the expert, the $\mathrm{k}$ coefficient can be changed. In our case we determine that up to 20 singularities, the value starts to become constant $(\mathrm{k}=1.1)$.

$$
u_{n b}=k^{-\left(N B_{s}\right)} \text { with } k=1.1
$$

\subsection{Quality determination by data fusion}

In order to determine the quality product, we merge singularity impacts and the singularity number criterion. There are three kinds of merging operators (Bloch, 1996):

- The severe operators which satisfy $F(a, b) \leq \operatorname{Min}(a, b)$

- The indulgent operators which satisfy $F(a, b) \geq \operatorname{Max}(a, b)$

- The compromise operator which satisfy $a \leq F a, b \leq b$, if $a \leq b$ $b \leq F a, b \leq a$, if $b \leq a$

In the quality evaluation, Expert never evaluates products on the best singularities. So indulgent operators cannot be used. The two others operators translate different visions from the expert in the quality evaluation. We propose to compare different operators which appear us to be well adapted to the used and Expert quality evaluation.

The first operator which can be used is the operator defined by Perez-Orama in (Perez Oramas, 2000) and describe by 13 . This operator (PO) is a compromise operator when the minimum value is under 0.5 , otherwise it is an indulgent operator. This characteristic can be interesting to isolate product with few singularities and evaluate the worst product.

$$
F(a, b)=\operatorname{Min}\left(1, \frac{\min (a, b)}{1-\operatorname{Min}(a, b)}\right)
$$

The Hamacher operator, described in 14 , is a severe operator. That means this operator gives result under the worst singularity. This can be useful to evaluate quality for product where visual quality is very important (joinery, cabinet, ...) because only products with high quality are highlighted.

$$
F(a, b)=\frac{a b}{a+b-a b}
$$

The Ordered Weight Average(OWA) adapted to our situation too, described in 15 , is a compromised operator. Usually the product quality is based on a part of the worst singularities (represented by the $\alpha$ which represent the percent of product fusion). The OWA allows to attribute weight only on this part of the singularities impact $C_{i}$.

$$
\begin{gathered}
F\left(C_{1}, \ldots C_{n}\right)=\sum_{i=1}^{n} w_{i} C_{i} \\
\text { and } C_{1} \geq C_{2} \geq \ldots \geq C_{n-1} \geq C_{n} \\
\text { with }\left\{\begin{array}{c}
w_{i}=0, \forall i \in[1,\lfloor\alpha n\rfloor] \\
w_{i}=\frac{1}{\alpha * N B_{s}} \forall i \in[\lceil\alpha n\rceil, n]
\end{array}\right.
\end{gathered}
$$


The results provided by these three operators will be compared to the arithmetic mean (used as a benchmark) which is a classical operator when the aggregation comportment is unknown.

\subsection{Operators comparison by the use of real case study}

The proposed methodology is then applied on the batch described in the table 5. These data is taken in into our partner sawmill. The first column indicates the piece number, the second the criterion $R_{n b}$ (function of the number of singularities), the third, all the singularities impact present on the product and the last the product aesthetics class gives by the sawmill Expert. The aim is to compare some fusion operators to the expert vision so as to find the closest.

Table 5: Pieces used to compare fusion operators.

\begin{tabular}{|c|c|c|c|c|c|c|}
\hline \multirow{2}{*}{$\frac{\text { Prod }}{\mathrm{a}}$} & \multirow{2}{*}{$\frac{R_{n b}}{0.91}$} & \multicolumn{4}{|c|}{$C_{u}$} & $\mathrm{Q}$ \\
\hline & & \multicolumn{4}{|l|}{0.888} & 0 \\
\hline $\mathrm{b}$ & 0.91 & 0.439 & & & & 1 \\
\hline \multirow[t]{3}{*}{$\mathrm{c}$} & \multirow[t]{3}{*}{0.42} & 0.888 & 0.866 & 0.826 & 0.776 & 1 \\
\hline & & 0.748 & 0.746 & 0.674 & 0.601 & \\
\hline & & 0.520 & & & & \\
\hline $\mathrm{d}$ & 0.75 & 0.814 & 0.740 & 0.259 & & 1 \\
\hline $\mathrm{e}$ & 0.75 & 0.888 & 0.372 & 0.332 & & 2 \\
\hline \multirow[t]{2}{*}{$\mathrm{f}$} & \multirow[t]{2}{*}{0.51} & 0.814 & 0.740 & 0.694 & 0.601 & 2 \\
\hline & & 0.565 & 0.432 & 0.312 & & \\
\hline \multirow[t]{2}{*}{ g } & \multirow[t]{2}{*}{0.51} & 0.725 & 0.667 & 0.587 & 0.479 & 3 \\
\hline & & 0.423 & 0.372 & 0.332 & & \\
\hline
\end{tabular}

- Piece 1: only one singularity with few impact (value close to 1), used for cabinet work.

- Piece 2: only one singularity with high impact,used for joinery work.

- Piece 3: lot of singularity with few impact, function of the use, the quality can be high or not thanks to the singularities number. in our case the expert class the product for joinery.

- Piece 4 and 5: the same number of singularities but one with more singularities with few impact (4) used for joinery work and the other the opposite (5) used in industrial carpentry.

- Piece 6 and 7: more singularities than 4 and 5 . Used respectively in industrial carpentry and traditional carpentry.

To compare the operators, two features are studied: the products ranking (cf. table 6) and the distance between them (cf. figure 6). Operators have to be compared on the distance between ranked product and the products groups they do. If the ranking is good but groups are totally different from the Expert choice (two products by in the same aesthetic class on the Expert judgement have to be close one to the other) the operator is less efficient than an operator which wrong ranks but keeps the right group of products.

Table 6: Piece ranking for each fusion operator.

\begin{tabular}{c|c|c|c|c|c|c} 
Rank & Exp & Hama & $\begin{array}{r}\text { OWA } \\
(\alpha=.2)\end{array}$ & $\begin{array}{r}\text { OWA } \\
(\alpha=.8)\end{array}$ & PO & Mean \\
\hline 1 & $\mathrm{a}$ & $\mathrm{a}(.82)$ & $\mathrm{a}(.89)$ & $\mathrm{a}(.90)$ & $\mathrm{a}(1)$ & $\mathrm{a}(.90)$ \\
2 & $\mathrm{~b}$ & $\mathrm{~b}(.42)$ & $\mathrm{c}(.47)$ & $\mathrm{b}(.67)$ & $\mathrm{b}(.43)$ & $\mathrm{c}(.71)$ \\
3 & $\mathrm{c}$ & $\mathrm{c}(.05)$ & $\mathrm{b}(.44)$ & $\mathrm{c}(.66)$ & $\mathrm{c}(.43)$ & $\mathrm{b}(.67)$ \\
4 & $\mathrm{~d}$ & $\mathrm{e}(.04)$ & $\mathrm{f}(.37)$ & $\mathrm{d}(.64)$ & $\mathrm{e}(.31)$ & $\mathrm{d}(.64)$ \\
5 & $\mathrm{e}$ & $\mathrm{c}(.0)$ & $\mathrm{g}(.35)$ & $\mathrm{e}(.59)$ & $\mathrm{g}(.31)$ & $\mathrm{e}(.59)$ \\
6 & $\mathrm{f}$ & $\mathrm{f}(.0)$ & $\mathrm{e}(.33)$ & $\mathrm{f}(.55)$ & $\mathrm{f}(.29)$ & $\mathrm{f}(.58)$ \\
7 & $\mathrm{~g}$ & $\mathrm{~g}(.0)$ & $\mathrm{d}(.26)$ & $\mathrm{g}(.48)$ & $\mathrm{d}(.23)$ & $\mathrm{g}(.51)$
\end{tabular}

The Hamacher operator, as it is the only pessimist operator, gives the lowest results. This operator his very efficient to highlight product which have good features. When there is a lot of singularities, this operator reaches towards highly downgrade product (up to 2 singularities, maximum value is 0.5 ). Three groups of product are made: $(\mathrm{a}, \mathrm{b})$ on the top as the expert, $(\mathrm{e}, \mathrm{d})$ and $(\mathrm{c}, \mathrm{f}, \mathrm{g})$ on the low. These seconds two groups mix quality product express by the Expert.So this operator is useful to evaluate high quality products.

The Perez-Orama operator gives high importance to product with all singularity impacts up to 0.5 and downgrade the others. It assumes that products with less than 8 singularities with an impact up to 0.5 , have a quality equal to 1 . This operator is particularly useful for a first ranking and extracts products described above (less than 8 singularities with impact up to 0.5 ). The operator place on opposite ends a and $\mathrm{d}$ and does two groups, $(b, c)$ and $(e, f, g)$. This classification is close to the expert choice (apart for d). This operator is efficient to class low quality products.

The $\alpha$ factor in the OWA operator may change value. We propose to compare $\alpha=0.2$ and $\alpha=0.8$. In the $\alpha=0.2$ case, the ranking gives, as the PO operator, four classes ((a),(c,b),(e,f,g,) and (d)) and the same observation as the previous operator. In the $\alpha=0.8$ case, the ranking is the same than the expert one. Moreover, product groups provided by this operator are the same than the expert classification ((a), (b,c,d),(e,f),(g)). Classes are close to one another but allowed to classify product as the expert estimation. The Expert which choose this ranking should have a decision process following this vision.

The last is the mean operator. It gives different ranking than the expert ranking but the products groups are respected. This operator may be used to group product with the same features without respect the ranking. This behaviour is interesting for carpentry product for which the ranking is not important. 


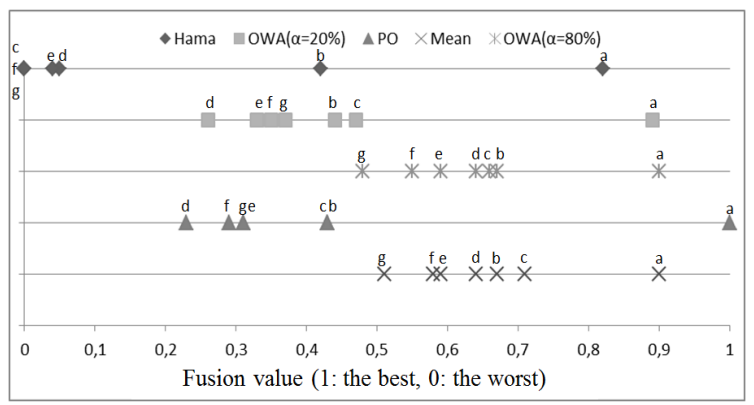

Figure 6: Product ranking for each operator

\section{CONCLUSIONS}

In this article, we present a way to determine the product quality in the wood industry. We decided to base the product quality evaluation on the singularities impact. As the information used to determine the singularity impact and the quality product are uncertain, imprecise, imperfect, we have to use operators which take into account of them.

The singularity impact is evaluated on criteria which are linked by interaction. Moreover, the poorness of the sample and the knowledge on the process decision, lead us to use the Choquet integral to determine impact. The quality determination is done by merging the singularity impact and the number of singularities. The use of different operators allows us to cover the majority of cases concerning the product quality determination. The comparison with the expert ranking and classification allows to conclude OWA operator with $\alpha=0.8$ reflects his choice.

This quality have a virtual nature. Moreover it is expressed with a regression value while Experts use linguistics quality classes. To take into account the virtual nature and the linguistics class representation, a fuzzyfication step may be used to obtain the belonging to quality classes as expressed by Expert.

All of that allows to conclude to the pertinence of our proposition. In perspective, other decision ways will be used and in particular the evaluation of the quality from the singularity characteristics (only one step). To do so, other fusion operators will be used as the Fuzzy Rule Classifier or classification operators.

\section{ACKNOWLEDGEMENTS}

The authors gratefully acknowledge the financial support of the CPER 2007-2013 Structuration du Pôle de Compétitivité Fibres GrandEst (Competitiveness Fibre Cluster), through local (Conseil Général des Vosges), regional (Région Lorraine), national (DRRT and
FNADT) and European (FEDER) funds.

\section{REFERENCES}

Almecija, B., Bombardier, V., and Charpentier, P. (2012). Modeling quality knowledge to design log sorting system by $\mathrm{x}$ rays tomography. In Information Control Problems in Manufacturing, volume 14, pages 11901195.

Bloch, I. (1996). Information combination operators for data fusion: A comparative review with classification. Systems, Man and Cybernetics, Part A: Systems and Humans, IEEE Transactions on, 26(1):52-67.

Bombardier, V., Mazaud, C., Lhoste, P., and Vogrig, R. (2007). Contribution of fuzzy reasoning method to knowledge integration in a defect recognition system. Computers in industry, 58(4):355-366.

Bucur, V. (2003). Techniques for high resolution imaging of wood structure: a review. Measurement Science and Technology, 14(12):R91.

Choquet, G. (1953). Theory of capacities. In Annales de l'institut Fourier, volume 5, page 87.

Dupuy, C. (2004). Analyse et conception d'outils pour la traçabilité de produits agroalimentaire afin d'optimiser la dispersion des lots de fabrication. $\mathrm{PhD}$ thesis, Institue National des Sciences Appliquées de lyon.

Grabisch, M., Kojadinovic, I., and Meyer, P. (2008). A review of methods for capacity identification in choquet integral based multi-attribute utility theory: Applications of the kappalab $r$ package. European journal of operational research, 186(2):766-785.

Grabisch, M. and Labreuche, C. (2008). A decade of application of the choquet and sugeno integrals in multicriteria decision aid. 4OR: A Quarterly Journal of $O p$ erations Research, 6(1):1-44.

Guillot, J., Lanvin, J., and Sandoz, J. (1996). Classement structure du sapin/épicéa par réseaux neuronaux à partir des mesures sylvatest. In Sciences et industries du bois. Colloque, pages 377-384.

Jover, J., Thomas, A., and Bombardier, V. (2011). Marquage du bois dans la masse : intérêts et perspectives. In $9 e$ Congrès International de Génie Industriel, CIGI 2011, St Sauveur, Canada.

Marichal, J.-L. and Roubens, M. (2000). Determination of weights of interacting criteria from a reference set. European journal of operational Research, 124(3):641-650.

PEFC1 (2010). Pefc st 1003:2010: Sustainable forest management.

Perez Oramas, O. (2000). Contribution une méthodologie d'intégration de connaissances pour le traitement d'images. Application à la détection de contours par régles linguistiques floues. $\mathrm{PhD}$ thesis, université Henry Poincaré.

Todoroki, C. and Rönnqvist, M. (2002). Dynamic control of timber production at a sawmill with log sawing optimization. Scandinavian Journal of Forest Research, 17(1):79-89. 